\title{
A Study to Assess the Impact of SOFA Scoring on Mortality and Morbidity in Patients with Surgical Sepsis at PESIMSR, Kuppam
}

\author{
Yugavardhan $\mathbf{N}^{1}$, Shivananda ${ }^{2}$, Tejswaroop $\mathrm{P}^{3}$, Sri Valli $\mathbf{V}^{4}$ \\ ${ }^{1}$ Senior Resident, Department of General Surgery, ${ }^{2}$ Professor of Surgery, Department of General Surgery, ${ }^{3}$ Post Graduate \\ Student, Department of General Surgery, ${ }^{4}$ Post Graduate Student, Department of General Surgery, PES Institute of Medical \\ Sciences and Research (PESIMSR), India
}

Corresponding author: Dr. Tejswaroop P, Post Graduate Student, Department of General Surgery, PES Institute of Medical Sciences and Research (PESIMSR), India

DOI: http://dx.doi.org/10.21276/ijcmsr.2019.4.3.23

How to cite this article: Yugavardhan N, Shivananda, Tejswaroop P, Sri Valli V. aA study to assess the impact of sofa scoring on mortality and morbidity in patients with surgical sepsis at PESIMSR, Kuppam. International Journal of Contemporary Medicine Surgery and Radiology. 2019;4(3):C102-C105.

\section{A B S T R A C T}

Introduction: The commonest cause of death in surgical patients is sepsis. SIRS (Systemic Inflammatory Response Syndrome) triggers of a series of events, ultimately evolving into shock and MODS, resulting in early morbidity and mortality, if timely intervention does not take place. The present study designed to assess the effect and degree of organ dysfunction on the morbidity and mortality of patients with surgical problems using a scoring system such as SOFA scoring.

Material and Methods: Present study was cross sectional study conducted among patients, admitted in the PES Institute of Medical Sciences and Research (PESIMSR), SICU presenting with sepsis at the time of first examination between November 2015 to October 2017.

Results: A total of Eighty patients were included in the study. Majority of the patients are in age group $>50$ years and $67.5 \%$ were males. Among eighty patients twenty five patients are expired, seven patients got discharged against medical advice. Highest SOFA was noted on day 3 , was significantly higher in the expired patients when compared in survived patients in all the 5 days.

Conclusion: Higher SOFA score is a predictor of mortality and morbidity in patients with surgical sepsis. SOFA score is a simple, but effective prognostic indicator and evaluator for patient progress in ICU.

Keyswords: Surgical Sepsis, SIRS (systemic Inflammatory Response Syndrome), MODS (Multiorgan Dysfunction Syndrome), Sequential Organ Failure Assessment Score (SOFA), PESIMSR, Kuppam

\section{INTRODUCTION}

Hippocrates first introduced the term sepsis with the word 'sepidon,' which means 'distortion, dissolution of a web structure' between 460-370 BC Sepsis being a common problem, may ultimately evolve into shock and Multiple organ dysfunction syndrome resulting in early morbidity and mortality among surgical patients. ${ }^{1,2}$

In the United States, over 970,000 sepsis cases are admitted annually, and the numbers have been rising year over year . incidence of sepsis among hospitalized patients is around $8.7 \%$ per year. Additionally, sepsis accounts for more than $50 \%$ of hospital deaths., ${ }^{3,4}$ Data from India are sparse and in the form of epidemiology of infection. ${ }^{5}$ Severe sepsis is common in Indian ICUs and is mainly due to Gramnegative organisms. ICU mortality is high due to increased length of stay. ${ }^{6}$

Sepsis represents the clinical response to non- specific insult of either infectious or non- infectious origin., ${ }^{7,8}$ This host response is defined by the Society of Critical Care Medicine as Systemic Inflammatory Response Syndrome(SIRS). ${ }^{9}$ SIRS if left unchecked, progresses to multiple organ dysfunction syndrome..$^{10,11}$

One of the main barriers to early interventions in sepsis is the lack of diagnostic tools and this is compounded by the fact that sepsis is a heterogeneous and enigmatic syndrome with no gold standard for diagnosis. ${ }^{12,13}$ Risk predictor based on scoring system has emerged as new way for early prediction to prevent from Multiple Organ Dysfunction Score . Types of Scoring Systems available are APACHE, SAPS, MPM, SOFA and MODS (Multiple Organ Dysfunction Score). No scoring system currently incorporates all these features, hence we have selected the scoring system most applicable to the Indian ICU setup the SOFA Score. The SOFA (Sequential Assessment of Organ Dysfunction) system was created in a consensus meeting of the European Society of Intensive Care Medicine in 1994 and further revised in 1996. ${ }^{14,15}$

The present study was designed to assess the effect and degree of organ dysfunction on the morbidity and mortality of patients with surgical problems using a scoring system such as SOFA scoring.

\section{MATERIAL AND METHODS}

Present study was cross sectional study conducted among 
patients, admitted in the PES Institute of Medical Sciences and Research (PESIMSR), SICU presenting with sepsis at the time of first examination between November 2015 to October 2017.

Sample Size: Considering the proportion of $71 \%$ surgical sepsis in ICU admissions with $10 \%$ permissible error (Absolute Precision), sample size turns out to be $80 . .^{15}$ $\mathrm{n}=\mathrm{Z}^{2} \mathrm{P}(\mathrm{P}-\mathrm{q}) / \mathrm{L} 2=(1.96)^{2} * 71 * 29 / 10 * 10=79.09$ The minimum sample size required for the study is a Sample of 79.09 which is rounded to 80 .

Adult patients with surgical problems (operative or non operative) in the SICU were included in the study, a total of 80 patients were included under our study. Data was collected in predesigned proforma with the informed and written consent of the patient and if the concerned person was not able to give consent then the consent from close relatives was taken.

Inclusion criteria: Adult patients of more than 18 years with surgical problems (operative or non-operative) and those who showed the features of sepsis/shock/ MODS including unconscious/comatose patients .

Exclusion criteria: Children $<18$ years . Patients admitted in general wards and Patient who expired within two days of ICU stay.

Method of data collection: History and clinical examination, Blood investigations, relevant body fluid Cultures was obtained. The parameters involved in the SOFA scoring will be followed up on a daily basis for five days.

The SOFA is a six-organ dysfunction/failure score measuring multiple organ failure daily. This scoring system is based on six different scores, one each for the respiratory, cardiovascular, hepatic, coagulation, renal and neurological systems. Each organ is graded from 0 (normal) to 4 (the most abnormal), providing a daily score of 0 to 24 points. Calculation of SOFA score during the first few days of ICU admission is a good indicator of prognosis. Both the mean and highest SOFA scores are particularly useful predictors of outcome. Independent of the initial score, an increase in SOFA score during the first 48 hours in the ICU predicts a mortality rate of at least $50 \%{ }^{15}$

\section{STATISTICAL ANALYSIS}

All results was recorded and tabulated and analyzed using Microsoft excel spread sheet ver 2013. Quantitative variables were described in the form of mean and standard deviation. Qualitative variables were described in the form of frequency and percentage. Inferential statistics was calculated using unpaired t test, Chi square tests. A $P$ value of 0.05 or less was considered as statistically significant.

\section{RESULTS}

Majority of patients belong to $51-70$ years with a mean age of $53 \pm 16$ years. Least were below 30 years of age group . Males constitute about $67.5 \%$ and females constitute $32.5 \%$ of the total patients. Minimum age of the patient is 19 years and maximum age is 90 years (table- 1 ).

Among 80 patients who were included in the study, majority

\begin{tabular}{|l|c|c|}
\hline Age in years & Number of patients & Percentage \\
\hline$<30$ & 8 & 10.0 \\
\hline $31-50$ & 24 & 30.0 \\
\hline $51-70$ & 35 & 43.75 \\
\hline$>70$ & 13 & 16.25 \\
\hline TOTAL & 80 & 100.0 \\
\hline \multicolumn{3}{|c|}{ Table-1: Age distribution of study subjects } \\
\hline
\end{tabular}

\begin{tabular}{|l|c|c|c|}
\hline Day & $\begin{array}{c}\text { Survived } \\
\mathbf{N}=\mathbf{4 8}\end{array}$ & $\begin{array}{c}\text { Expired } \\
\mathbf{N = 2 5}\end{array}$ & P value \\
\hline Day 1 & $5.56 \pm 2.70$ & $7.2 \pm 3.01$ & 0.0707 \\
\hline Day2 & $5.83 \pm 2.95$ & $8.68 \pm 3.02$ & 0.0018 \\
\hline Day3 & $5.95 \pm 2.97$ & $8.76 \pm 2.58$ & 0.0016 \\
\hline Day4 & $5.58 \pm 2.67$ & $8.88 \pm 2.75$ & 0.0005 \\
\hline Day 5 & $5.30 \pm 2.65$ & $8.70 \pm 3.51$ & 0.0020 \\
\hline \multicolumn{4}{|c|}{ Table-2: Sofa Score In Comparison With Survived, Expired } \\
\hline
\end{tabular}

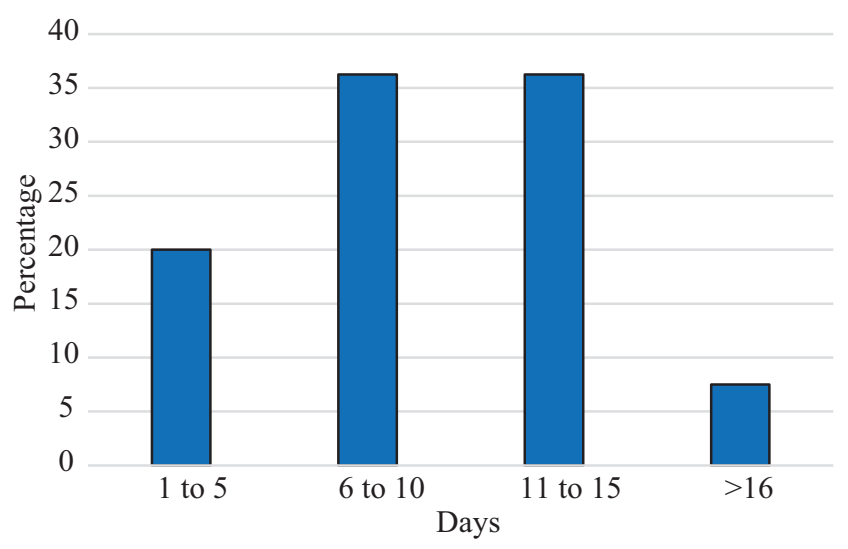

Figure-1: Duration of hospital stay.

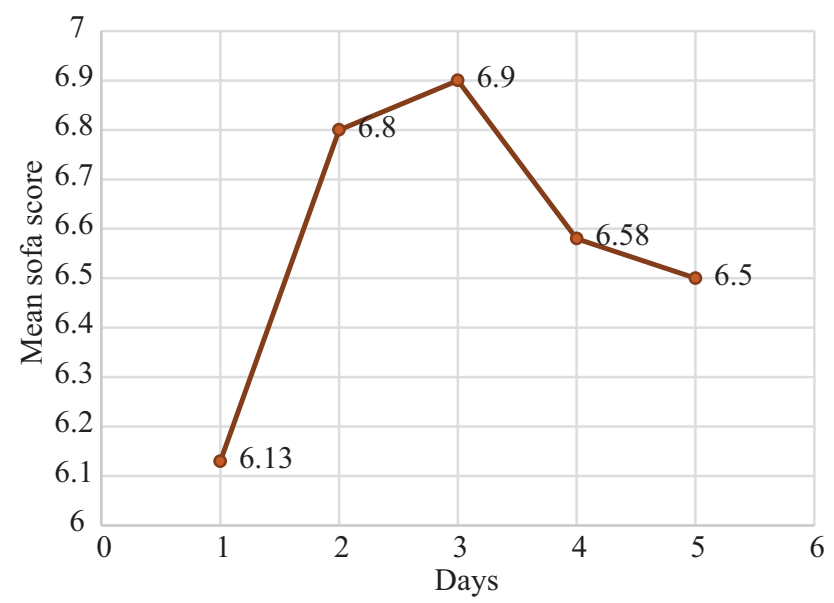

Figure-2: Evaluation of SOFA score

were Exploratory Laparotomies secondary to Hollow viscus perforation, Diabetic foot and Necrotizing fasciitis. Other cases which were included in this study were Liver abscess, Necrotising pancreatitis, carcinoma -caecum, sigmoid colon, stomach, rectum and head of pancreas, Acute Intestinal obstruction, Obstructed Hernias and Appendicular peroration.

More than $80 \%$ of the patients stayed more than 5 days in hospital and $45 \%$ of patients stayed more than 5 days in ICU. 
$34 \%$ of patients stayed for 4 to 5 days and $21 \%$ for less than 4 days (figure-1).

Among the 80 patients in the study $55 \%$ of the patients were diabetic patients, $17.5 \%$ of the patients were Hypertensive patients and remaining suffered from Ischemic Heart Disease, Osteoarthritis, Tuberculosis, Bronchial Asthma, COPD and RHD. Among the 80 patients in the study 48 patients survived, 25 patients expired, 7 patients got discharged against medical advice.

In all patients, the maximum sofa score was noted on day 3 (6.9 \pm 3.1 ) followed by day 2 i.e $6.8 \pm 3.34$ followed by sofa score on day 4 is $6.58 \pm 3.07$ (figure-2).

Sofa score was significantly higher in the expired patients when compared in survived patients in all the 5 days which says that SOFA score is a good predictor for Sepsis related mortality (table-2).

\section{DISCUSSION}

In the present study mean age of study population is $53 \pm 16$ years. $90 \%$ of patients are in group of $>30$ years and $60 \%$ of patients of $>50$ years . Similar findings were observed in study done by R.Moreno J.-L.Vincent et al i.e the mean age of the patient is $55 \pm 19$ years ranging from $12-95$ years. ${ }^{16}$ In a study done by Aditi Jain et al where more than $80 \%$ of subjects were $<55$ years of age $.67 .5 \%$ of the surgical cases in our study were male patients. ${ }^{17} \mathrm{~A}$ study done by Takeshi Umegaki et al showed over half of the patients were males (61.4\%). ${ }^{18} \mathrm{~A}$ study done by Aditi Jain et al, SOFA score was determined $24 \mathrm{~h}$ postadmission to ICU and subsequently every $48 \mathrm{~h}$ for the first 10 days. Patients were followed till discharge/death/ transfer from the ICU. Initial SOFA score, highest and mean SOFA scores were calculated and correlated with mortality and duration of stay in ICU. The mortality rate was 39\% . The maximum score in survivors $(3.92 \pm 2.17)$ was significantly lower than nonsurvivors $(8.9 \pm 3.45)$. The initial SOFA score had a strong statistical correlation with mortality. ${ }^{17} \mathrm{~A}$ study done by R.Moreno J.-L.Vincent et al, the mean total maximum SOFA score was $8.2+5.4$ points, median 7 points, range $0 \pm 24$ points, and was significantly higher in nonsurvivors than in survivors $13.6 \pm 4.8$ points versus $6.7 \pm 4.5$ points, $\mathrm{p}<0.001) .{ }^{16}$ A Study done by Flavio lopes Ferreira et al, for more than 24 hours SOFA score was calculated. Independent of the initial score, an increase in sofa score during the first $48 \mathrm{hrs}$ in ICU predicts a mortality rate of atleast $50 \%$.He concluded that SOFA score during the first few days of ICU admission is a good indicator of prognosis. ${ }^{19}$ The above findings were in consensus with this study the mean sofa score was significantly higher in non-survivors than in survivors and which is statistically significant . In all patients, the maximum sofa score was noted on day 3 which corresponds to maximum mortality . which suggests the patients remaining in the ICU for atleast 72 hours are at an increased risk for mortality after discharge.

Conclusion: SOFA score is a simple, but effective prognostic indicator and evaluator for patient progress in ICU. Higher SOFA score is a predictor of mortality and morbidity in patients with surgical sepsis. Day 1 SOFA can triage the patients into risk categories. For further management, mean and maximum score help determine the severity of illness and can act as a guide for the intensity of therapy required for each patient.

\section{REFERENCES}

1. Geroulanos S, Douka ET. Historical perspective of the word “sepsis". Intensive Care Med. 2006; 32:p 2077.

2. Johnson GB, Brunn GJ, Samstein B, Platt JL. New insight into the pathogenesis of sepsis and the sepsis syndrome. Surgery. 2005;137(1):393-5.

3. Liu V, Escobar GJ, Greene JD, et al. Hospital deaths in patients with sepsis from 2 independent cohorts. JAMA 2014; 312(3):90-92.

4. Martin GS. Sepsis, severe sepsis and septic shock: Changes in incidence, pathogens and outcomes. Expert Rev Anti Infect Ther 2012; 109(5):701-706.

5. Ghanshani RV, Gupta R, Sood S, Bansal A, Joad SH, Khedar RS. Epidemiology of infections in a medical ICU in India. Intensive Care Med. 2014;40(2):456-7.

6. Sharmila Chatterjee, Mahuya Bhattacharya, and Subhash Kumar Todi. Epidemiology of Adultpopulation Sepsis in India: A Single Center 5 Year Experience. Indian J Crit Care Med. 2017; 21(9): 573577.

7. Levy Mitchell M, Fink Mitchell P, Marshall John C, Abraham Edward Angus, Derek Cook Deborah, Cohen Jonathan Opal Et al. Critical Care Medicine. 2003;31(4): 1250-6.

8. Jackson AJ, Coats p, Nixon GE, Orr DJ, Teenan RP, Wadsworth RM. Innate immune pathways in neointimal hyperplasia formation: a role for Toll-like receptor 4. BJS 2009; 96 (s1): 2.

9. Rangel-Fausto MS PD, Costigan M. The natural history of the systemic inflammatory response syndrome (SIRS) . A prospective study, JMMA. 1995; 273(6): 117-213

10. Selberg O, Hecker H, Martin M et al. Discrimination of sepsis and systemic inflammatory response syndrome by determination of circulating plasma concentration of procalcitonin, protein complement $3 \mathrm{a}$ and interleukin-6. Crit Care Med. 2000; 28(3): 2793-8.

11. Deitch AE. Multiple organ Failure. Pathophysiology and potential future therapy, Ann Surg. 1992; 216(1): 117-134.

12. Churpek MM,Zadravecz FJ, Winslow C, et al.Incidence and prognostic value of the systemic inflammatory response syndrome and organ dysfunctions in ward patients. Am J Respir Crit Care Med 2015;192(5):95864.

13. Kaukonen KM, Bailey M, Pilcher D, et al. Systemic inflammatory response syndrome criteria in defining severe sepsis. N Engl J Med. 2015;372(4):1629-38.

14. Abraham, Edward; Singer, Mervyn. Mechanisms of sepsis- induced organ dysfunction. Critical Care Medicine. 2007;35(10): 2408-16.

15. Christopher Bouch D, Jonathan Thompson P. Severity scoring systems in the critically ill .Oxford Journalsmedicine-BJA.2008; 8(5):181-185.

16. Moreno R, Vincent JL, et al . The use of maximum SOFA score to quantify organ dysfunction/failure in intensive care. Results of a prospective, multicentre study . Intensive Care Med. 1999;25(7):686-96.

17. Jain A, Palta S, Saroa R, Palta A, Sama S, Gombar 
S. Sequential organ failure assessment scoring and prediction of patient's outcome in Intensive Care Unit of a tertiary care hospital. Journal of Anaesthesiology, Clinical Pharmacology. 2016;32(3):364-368.

18. Umegaki T,ikai H, Imanaka $Y$. The impact of acute organ dysfunction on patients mortality with severe sepsis .J Anaesthesiol clin pharmacol 2011;27(3):180-4.

19. Flavio Lopes Ferreira, Daliana Peres Bota, Annette Bross, Christian Mélot, Jean-Louis Vincent. Serial Evaluation of the SOFA Score to Predict Outcome in Critically Ill Patients.JAMA. 2001;286(14):1754-1758.

Source of Support: Nil; Conflict of Interest: None

Submitted: 30-05-2019; Accepted: 22-07-2019; Published online: 19-08-2019 